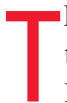
he excessive cardiovascular morbidity and mortality associated with diabetes (sometimes termed "the burden of heart disease in diabetes") has been recognised for a long time. Because of this, I have suggested that diabetes should be defined as a state of premature cardiovascular death which is associated with chronic hyperglycaemia and may also be associated with blindness and renal failure. This was first placed in context by the Framingham study, where middle aged people with diabetes had an increased coronary heart disease morbidity and mortality that could not be explained by the traditional cardiovascular risk factors of smoking, age, raised cholesterol, raised blood pressure, or obesity. ${ }^{1}$ This suggested a possible unique role for diabetes as a risk factor for the development of cardiovascular disease. Women with diabetes had the same prevalence of cardiovascular problems as men with diabetes, greatly increasing the risk compared to non-diabetic women, leading to the phrase "women with diabetes loose the protection of their gender". An excess of congestive cardiac failure was also noted that could not be explained by the presence of coronary heart disease, adding support to the existence of a possible "diabetic cardiomyopathy".

From a critical perspective, however, this study was guilty of many of the problems that have affected research in this area ever since. A combination of patients with type 1 and type 2 diabetes was studied, and no attempt was made to classify the type of diabetes in an individual subject. The diagnostic criteria for diabetes were not consistent within the various publications from the Framingham study, leading to differing numbers of patients with diabetes in different publications. The number of subjects with diabetes was very small, which may have exaggerated the risks of some of the end points, such as cardiac failure in women with diabetes. The small number of subjects also means that the Framingham equation based on these data is much less exact for estimating cardiovascular risk in people with diabetes. Only middle aged and elderly subjects were studied, where the absolute risk is highest, and no attempt was made to study younger diabetic subjects, where the relative risk for people with diabetes is extraordinarily raised compared to non-diabetic subjects, yet the absolute risk remains relatively low.

A recent review by Timmis described considerations in the cardiological treatment of people with diabetes. ${ }^{2}$ This review examines the possible differing mechanisms of heart disease in people with type 1 and type 2 diabetes. The effects of measures to control blood glucose on vascular outcomes are described in these groups of patients. Finally, studies on the reduction of cardiovascular risk in diabetes by the treatment of hypertension and dyslipidaemia, including several newer publications in this area, are described in detail.

\title{
INSULIN RESISTANCE AND THE METABOLIC SYNDROME
}

Correspondence to: Dr Miles Fisher, Wards 4 \& 5, Glasgow Royal Infirmary, Glasgow, G4 OSF, UK; miles.fisher@northglasgow. scot.nhs.uk
The concept of insulin resistance was first introduced in the 1960s when it was noticed that patients following myocardial infarction had high insulin concentrations. Similar high insulin values were also seen in patients with hypertension, and this association was confirmed in many large, epidemiological studies. It was suggested that the pancreas increased the production of insulin to try and overcome the insulin resistance, and that this relative hyperinsulinaemia caused sodium retention and increased sympathetic nervous tone, so increasing the blood pressure.

In his 1988 Banting lecture, Reaven noted that resistance to insulin stimulated glucose uptake was present in the majority of patients with type 2 diabetes and impaired glucose tolerance, and in $25 \%$ of non-obese individuals with normal glucose tolerance. ${ }^{3}$ He suggested that glucose intolerance could be prevented if the beta cell was able to increase insulin secretion and maintain chronic hyperinsulinaemia. If this could not be achieved then decompensation of glucose tolerance occurred. He suggested that the relation between insulin resistance, hyperinsulinaemia, and glucose intolerance was mediated by changes in free fatty acid concentrations, as plasma free fatty acids could be reduced by small increments in insulin concentration. If hyperinsulinaemia could not be maintained, increased free fatty acids would lead to increased hepatic glucose production, and because of resistance to insulin stimulated glucose uptake, to hyperglycaemia. 
Reaven also identified a clustering of risk factors for coronary heart disease, including hyperinsulinaemia, impaired glucose tolerance, increased plasma triglyceride concentrations, decreased high density lipoprotein (HDL) cholesterol concentrations, and hypertension that he named "syndrome $X$ ".

Several other components of syndrome X or the "metabolic syndrome" have been added, including visceral adiposity, microalbuminuria, endothelial dysfunction, abnormal fibrinolysis, and markers of inflammation. It remains unclear if this is just a coincidental clustering of cardiovascular disease risk factors that are shared with risk factors for diabetes (the common soil hypothesis) or whether there is a single aetiological determinant as suggested by Reaven. The single determinant might be genetic, insulin resistance, visceral obesity, endothelial dysfunction, or inflammation, or there might be multiple interacting determinants.

A recent study from Italy measured insulin resistance using a homeostasis model assessment (HOMA), and found that insulin resistance was a strong independent predictor of cardiovascular disease in type 2 diabetes. ${ }^{4}$ This mathematical model utilises basal concentrations of glucose and insulin, and provides an index of relative insulin sensitivity. New oral hypoglycaemic treatments that target insulin resistance are described below, but insulin resistance is not at present a separate target for intervention in type 2 diabetes.

\section{HEART DISEASE AND TYPE 1 DIABETES}

Patients with type 1 diabetes tend to be younger than patients with type 2 diabetes (the average age of onset for type 1 diabetes is 14 years versus 60 years for type 2 diabetes). The pathophysiology of cardiovascular disease is probably different to that in type 2 diabetes, although recent studies suggest that many patients with type 1 diabetes become insulin resistant following the initiation of insulin treatment. As in the general population and in patients with type 2 diabetes, this may be explained by decreased physical activity and excessive food intake leading to visceral adiposity. This has been termed "double diabetes", but it is not certain if these are the only patients with type 1 diabetes that are at increased cardiovascular risk.

Several older studies suggested that the excessive risk was associated with varying degrees of renal impairment in patients with type 1 diabetes. Longstanding type 1 diabetes can also lead to autonomic neuropathy and a diabetic cardiomyopathy, ${ }^{5}$ and co-segregation of these problems in patients with poor glycaemic control is a possibility.

Recent studies have focused on new ways of cardiologically identifying patients with type 1 diabetes who have early cardiovascular problems, as the detection of proteinuria is not sufficiently sensitive to predict cardiovascular outcomes in type 1 diabetes, and investigation for autonomic neuropathy or the diabetic cardiomyopathy is not routinely performed. Estimation of the degree of coronary artery calcification using electron beam tomography is becoming increasingly accepted for risk stratification in the asymptomatic diabetic patient. ${ }^{6}$

\section{GLYCAEMIC CONTROL AND REDUCTION OF CARDIOVASCULAR RISK Type 1 diabetes}

The landmark trial of tight glycaemic control in people with type 1 diabetes was the diabetes control and complications trial (DCCT). ${ }^{7}$ Tight control, based on a continuous subcutaneous insulin infusion or basal bolus insulin regimen, was associated with a significant reduction in the development or progression of microvascular diabetic complications, including diabetic retinopathy and diabetic nephropathy, on a mean of 6.5 years of follow up. Careful analysis was performed of cardiovascular end points in the DCCT, including acute myocardial infarction and death from cardiovascular disease. ${ }^{7}$ There were a very small number of cardiovascular events in the study, however, and no significant effect of tight glycaemic control on vascular outcomes was demonstrated. Four definite myocardial infarctions were recorded, and all occurred in the conventional treatment group. A subsequent meta-analysis based on the DCCT study but also including several smaller studies concluded that intensive insulin treatment reduced the number of total major macrovascular events, including myocardial infarction, but that mortality was not affected with intensive insulin treatment.

\section{Type 2 diabetes}

The landmark trial of tight glycaemic control in people with type 2 diabetes was the UK prospective diabetes study (UKPDS) in 4209 subjects with recently diagnosed diabetes. ${ }^{8}$ Compared to the DCCT there was a smaller separation between the intensive and control groups. In the DCCT the average HbAlc in the intensive group was $7.2 \%$ compared to $9.1 \%$ in the conventional group. In the UKPDS the figures were $7.0 \%$ and $7.9 \%$ for the intensive and conventional groups, respectively.

Tight control, based on either treatment with sulfonylureas or insulin, was associated with a significant reduction in the development of microvascular complications, including diabetic retinopathy and nephropathy, on a mean of 10 years of follow up. ${ }^{8}$ Myocardial infarction was the most common single end point and occurred in around $12 \%$ of all patients. The mortality from myocardial infarction was around 50\%. The risk of a myocardial infarction was 14.7 events per 1000 patient years in the intensive group, and 17.4 events per 1000 patient years in the conventional group, a non-significant difference $(p=0.052)$.

For overweight patients a further possible randomisation was to intensive treatment with metformin, which exerts its principal hypoglycaemic effect by suppressing basal hepatic glucose production. Somewhat to the surprise of the investigators this group of diabetic patients did best, with a significant reduction in myocardial infarctions, cardiovascular deaths, and total mortality compared to the conventional treatment group. ${ }^{9}$ Based on the results of the UKPDS the treatment of first choice for overweight patients with type 2 diabetes not controlled by dieting is metformin, with sulfonylureas reserved as first choice for the minority of patients that are not overweight or cannot tolerate metformin.

Recently, a newer class of oral hypoglycaemic agents, the thiazolidenediones or glitazones, has become available for the treatment of type 2 diabetes. They act by binding to the peroxisome proliferator-activated receptor $\gamma(\operatorname{PPAR} \gamma)$ in the nucleus of the cell. They sensitise the body to its own insulin, reducing insulin resistance and enabling peripheral tissues to increase the uptake of glucose from the plasma. These drugs lower blood sugar, and also reduce blood pressure, microalbuminuria, and increase HDL cholesterol concentrations. Body weight increases, and complex changes in other lipids are described. Preliminary studies have demonstrated reductions in concentrations of $\mathrm{C}$ reactive protein, and white blood cell count, ${ }^{10}$ suggesting the possibility of extended benefit beyond blood glucose lowering effects; this is being tested in 
Trial acronyms

AFCAPS/TexCAPS: Air Force/Texas Coronary Atherosclerosis Prevention Study

ALLHAT: Antihypertensive and Lipid-Lowering Treatment to prevent Heart Attack Trial

DAIS: Diabetes Atherosclerosis Intervention Study

DCCT: Diabetes Control and Complications Trial

HDS: Hypertension in Diabetes Study

HOPE: Heart Outcomes Prevention Evaluation study

HOT: Hypertension Optimal Treatment trial

HPS: Heart Protection Study

IDNT: Irbesartan Diabetic Nephropathy Trial

IRMA2: Irbesartan in Patients with Type 2 Diabetes and Microalbuminuria Study

LIFE: Losartan Intervention For Endpoint Reduction in Hypertension

LIPID: Long-Term Intervention with Pravastatin in Ischaemic Disease study

RENAAL: Reduction of Endpoints in NIDDM with the Angiotensin II Antagonist Losartan

4S: Scandinavian Simvastatin Survival Study

SHEP: Systolic Hypertension in the Elderly Program

Syst-Eur: Systolic Hypertension in Europe trail

UKPDS: United Kingdom Prospective Diabetes Study

VA-HIT: Veterans Affairs High-Density Lipoprotein

Cholesterol Intervention Trial

WOSCOPS: West of Scotland Coronary Prevention Study

several large randomised studies with cardiovascular outcomes in people with diabetes.

Two other drugs have recently become available for the treatment of type 2 diabetes. Like sulfonylureas, repaglinide and nateglinide work by stimulating pancreatic potassium channels, releasing insulin. They are short acting, have to be taken more than once daily to control blood glucose concentrations, and offer no distinct advantages over sulfonylurea treatment. Recent epidemiological analysis shows that the level of postprandial hyperglycaemia may be a better predictor of cardiovascular outcomes than a fasting blood glucose concentration. The rapid onset of action of these agents means that improvements in postprandial hyperglycaemia are demonstrated when these drugs are compared to older agents, but there is as yet no published evidence that the use of these agents leads to improvements in cardiovascular outcomes.

\section{RISK FACTOR REDUCTION \\ Blood pressure control}

Evidence of benefit in reducing blood pressure in people with diabetes has come from subgroup analysis of several large studies (table 1), and from a small number of studies conducted in specific groups of patients with diabetes. The hypertension in diabetes study (HDS) was nested within the UKPDS and therefore included subjects with hypertension and recently diagnosed diabetes. ${ }^{11}$ Tight control of blood pressure, based on treatment with captopril or atenolol, reduced microvascular (diabetic retinopathy) and macrovascular (stroke) complications compared to less tight control. Interestingly, the blood pressure attained in the tight control group was similar to that which gave maximum benefit for

diabetic subjects in the hypertension optimal treatment (HOT) study, using a felodipine based treatment regimen.

The more recent LIFE (losartan intervention for endpoint reduction in hypertension) study examined people with hypertension and ECG evidence of left ventricular hypertrophy, and compared a regimen based on losartan with a regimen based on atenolol. ${ }^{12}$ For the study as a whole, a significant reduction was seen in the primary composite with losartan compared to atenolol. In particular, there were significant reductions in the number of strokes, with insignificant effects on cardiovascular death and myocardial infarctions. By contrast, for the diabetic subgroup there was no significant reduction in strokes or myocardial infarctions, but significant reductions were demonstrated in cardiovascular deaths and total mortality when the group treated with losartan were compared with those treated with atenolol. ${ }^{12}$ The reasons for these differences are not clear.

Angiotensin receptor antagonists have also been studied in hypertensive patients with type 2 diabetes and varying degrees of diabetic nephropathy. The IRMA2 (irbesartan in patients with type 2 diabetes and microalbuminuria) study examined diabetic patients with microalbuminuria, which occurs in one third of patients with type 2 diabetes. ${ }^{13}$ Irbesartan was better than placebo in slowing the progression from microalbuminuria to overt proteinuria. For subjects with overt proteinuria, irbesartan and losartan were better than placebo and amlodipine respectively in the IDNT (irbesartan diabetic nephropathy trial) and RENAAL (reduction in endpoints in NIDDM with the angiotensin II antagonist losartan) studies in reducing the composite end point of doubling of serum creatinine, end stage renal disease, and death. ${ }^{14}{ }^{15}$ When the components of the composite were examined, there were benefits in the renal outcomes of end stage renal disease or doubling of serum creatinine, but no effect on death. Unfortunately, these studies were terminated at around 2.5 years because of the renal benefit, and there was no significant benefit in reducing a mixture of secondary cardiovascular outcomes. It remains uncertain whether these drugs will give cardiovascular benefit over and above any benefit of blood pressure lowering on renal function, as has been suggested for ramipril in the HOPE (heart outcomes prevention evaluation) study (see below).

In the recently published ALLHAT study (antihypertensive and lipid-lowering treatment to prevent heart attack trial), chlorthalidone based hypotensive treatment was if anything better than treatment based on enalapril or amlodipine. ${ }^{16}$ All three were useful, safe, and well tolerated, dismissing previous fears that amlodipine might be of less benefit in people with diabetes. Patients with diabetes will often need two or more hypotensive agents to reach treatment targets, and regimens can include a combination of diuretics, ACE inhibitors, angiotensin II receptor antagonists, $\beta$ blockers, and calcium channel blockers. For patients with either microalbuminuria or overt proteinuria an ACE inhibitor or an angiotensin II receptor antagonist should be included in the combination.

\section{HOPE study}

The interpretation of the results of the HOPE study remains controversial. Using a simple study design, over 9297 people at high cardiovascular risk, including 3577 diabetic patients, received ramipril or placebo. Ramipril lowered the risk of the primary composite end point of myocardial infarction, stroke or cardiovascular death, including significant reductions in 
Table 1 Major trials of blood pressure lowering treatment in diabetes

Key trials containing large numbers of subjects with diabetes

- ALLHAT: chlorthalidone, amlodipine, lisinopril, 33357 subjects, 12063 with diabetes

- HOT: felodipine, 18790 subjects, 1501 with diabetes

- LIFE: losartan, atenolol, 9193 subjects, 1195 with diabetes and left ventricular hypertrophy

- SHEP: 4736 subjects, 583 with diabetes and systolic hypertension

- Syst-Eur: 4695 subjects, 492 with diabetes and systolic hypertension

Major trials containing only subjects with diabetes

- IRMA2: irbesartan, 590 subjects with diabetes and microalbuminuria

- IDNT: irbesartan, 1715 subjects with diabetes and nephropathy

- RENAAL: losartan, amlodipine, 1513 subjects with diabetes and nephropathy

- UKPDS/HDS: atenolol, captopril, 1148 subjects with hypertension and newly diagnosed diabetes

See box for explanation of trial acronyms.

each of the components of the composite and in total mortality. Data on blood pressure responses were not collected in detail, so it is uncertain whether this reduction in risk was as a result of blood pressure lowering in a high risk group of patients, or whether this was due to extended effects of ACE inhibition. The results for the diabetic subjects were published separately. ${ }^{17}$ In addition to cardiovascular benefit, there was a significant reduction in the development of overt diabetic nephropathy. Unfortunately, there was infrequent sampling of the degree of proteinuria throughout the study, with a single sample at baseline, one year, and five years. There was no significant effect of ramipril on the development of new microalbuminuria, but there was a reduction in the progression of microalbuminuria as measured by the mean albumin/creatinine ratio.

Several post-hoc subgroup publications from the HOPE study provide useful information in people with diabetes. The degree of albuminuria was measured in $97 \%$ of patients in the HOPE study. Microalbuminuria was detected in $32 \%$ of diabetic subjects at baseline and 15\% of non-diabetic subjects, and was associated with the duration of diabetes, increasing HbAlc, hypertension, smoking, and left ventricular hypertrophy. ${ }^{18}$ Microalbuminuria increased the adjusted relative risk of major cardiovascular risk on subsequent follow up.

One tenth of the subjects in the study had renal insufficiency at baseline, as determined by a mild increase in serum creatinine concentration (patients with more severe

Table 2 Major trials of lipid lowering treatment in diabetes

Major trials containing large numbers of subjects with diabetes for secondary prevention

- 4S: simvastatin, 4444 subjects, 202 with diabetes

- CARE: pravastatin, 4159 subjects, 586 with diabetes

- LIPID: pravastatin, 9014 subjects, 782 with diabetes

- HPS: simvastatin, 20536 subjects, 13386 subjects with prior myocardial infarction or other coronary heart disease, 1981 with diabetes

VA-Hit: gemfibrozil, 2531 subjects, 627 with diabetes

Major trial containing large numbers of subjects with diabetes for primary prevention

- HPS: simvastatin, 7150 subjects with no prior coronary heart disease, 3982 subjects with diabetes

See box for explanation of trial acronyms.
Diabetes and atherogenesis: key points

- For patients with type 1 diabetes the excessive cardiovascular burden may only affect a small number of people, especially those with renal complications, and ways of identifying these people need to be refined

- For people with type 2 diabetes, the excessive cardiovascular burden is not completely explained by conventional cardiovascular risk factors, and novel risk factors may have an important role

- Treatment of glycaemia using older agents may be of limited benefit. The use of newer oral hypoglycaemic agents, that give some improvements in both traditional and novel risk factors, is currently being explored

- Aggressive treatment of dyslipidaemia and hypertension is of proven efficacy

degrees of renal impairment were excluded). ${ }^{19}$ The cumulative incidence of the primary outcome was higher in patients with renal insufficiency than in those without, emphasising the high cardiovascular event rate in patients with renal impairment. A particular benefit in the reduction in cardiovascular events with ramipril was demonstrated in diabetic subjects with a raised serum creatinine compared to other groups.

\section{Lipid lowering treatment}

People with type 2 diabetes have been well represented in the landmark trials of lipid lowering treatment for the secondary prevention of cardiovascular disease (table 2). Separate subgroup analysis for people with diabetes has been published for several of these trials. Figure 1 illustrates the benefit of lipid lowering treatment on the combined end point of cardiovascular death and non-fatal myocardial infarction in people with diabetes compared to non-diabetic subjects in the older statin trials. Generally, people with diabetes have obtained similar or greater relative risk reduction from the use of statins (or gemfibrozil), but because of the much greater event rate they have obtained a greater absolute benefit. In the heart protection study (HPS), the event rate (cardiovascular death, myocardial infarction, stroke, revascularisations) in the placebo group for diabetic subjects with prior coronary disease was an astonishing $37.8 \%$, which was the highest for any subgroup in the study. This was reduced to $33.4 \%$ by simvastatin $40 \mathrm{mg} .{ }^{20}$

By contrast, people with diabetes were not well represented in the older landmark primary prevention studies, and so subgroup analysis is not feasible. In the HPS there were nearly 4000 patients with diabetes and no prior coronary heart disease, and the results for these patients are contained within the principal publication in the Lancet. However, this group included some people with prior cerebrovascular or peripheral vascular disease. In subsequent correspondence, the authors indicated that 2912 patients with diabetes did not have any diagnosed occlusive vascular disease at entry, and the major vascular event rate was reduced from $13.5 \%$ to $9.3 \%$ by simvastatin in these diabetic subjects.

The typical lipid abnormalities that are seen in type 2 diabetes and the insulin resistance syndrome are an increase in triglycerides, a fall in HDL cholesterol, and no real increase in total or low density lipoprotein (LDL) cholesterol. This pattern of dyslipidaemia might be expected to respond better to treatment with fibrates than statins. There were a 


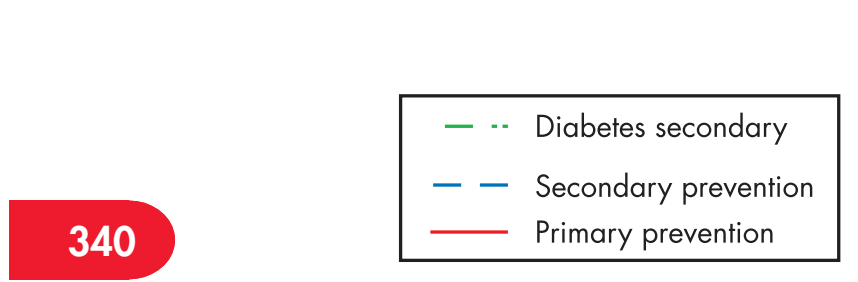

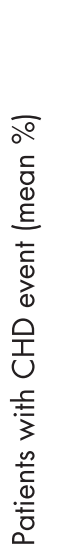
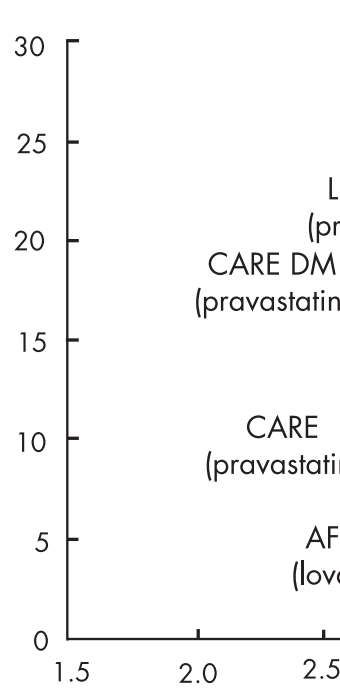

4S DM

\section{CARE DM}

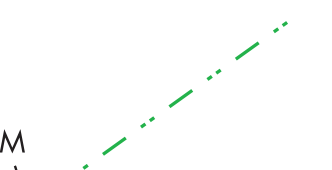

(simvastatin)

(placebo)

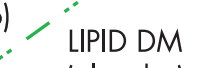

LIPID DM
(placebo)

CARE DM

LIPID DM

(pravastatin)

pravastatin)
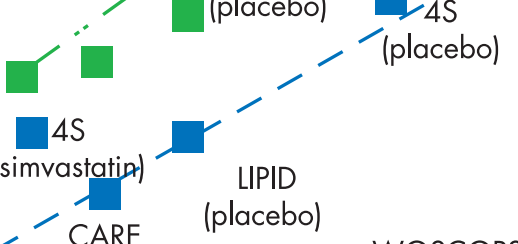

CARE (pravastatin)

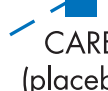

(placebo)

Placebo)

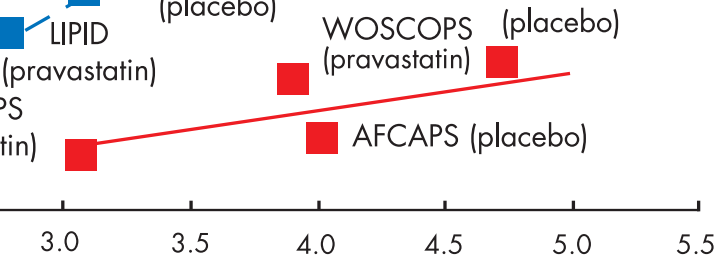

AFCAPS

(lovastatin)

3.5

4.0

Mean LDL cholesterol (mmol/I)

reasonable number of diabetic subjects in the VA-HIT study (Veterans Affairs high-density lipoprotein cholesterol intervention trial) of gemfibrozil, and separate subgroup analysis of diabetic subjects has recently been published. DAIS (diabetes atherosclerosis intervention study) was a study of the effects of fenofibrate in people with diabetes on surrogate angiographic markers. Fenofibrate was of some benefit in these subjects, but no difference was seen in harder cardiovascular outcomes. Studies are underway comparing the effects of fenofibrate compared to placebo on hard cardiovascular outcomes in patients with diabetes; in the longer term it is hoped to complete comparative studies of fibrates and statins on hard cardiovascular outcomes in people with diabetes.

\section{REFERENCES}

1 Kannel WB, McGee DL. Diabetes and cardiovascular disease. The Framingham study. JAMA 1979;241:2035-8.

- Key publication of the Framingham diabetes data based on 118 men and 121 women with diabetes among 5209 subjects.

2 Timmis AD. Diabetic heart disease: clinical considerations. Heart 2001;85:463-9.

3 Reaven GM. Role of insulin resistance in human disease. Diabetes 1988;37:1595-607.

- Review combining observed clustering of risk factors and hypothesis about free fatty acids as a major aetiological determinant.

4 Bonora E, Formentini G, Calcaterra F, et al. HOMA-estimated insulin resistance is an independent predictor of cardiovascular disease in type 2 diabetic subjects. Diabetes Care 2002;25:1135-41.

5 Fisher BM, Frier BM. Evidence for a specific heart disease of diabetes in humans. Diabet Med 1990:7:478-89.

6 Budoff MJ. Diabetic patients and coronary calcium: risk stratification, compliance, and plaque progression. Diabetes Care 2003;26:541-2.

7 The Diabetes Control and Complications Trial (DCCT) Research Group. Effect of intensive diabetes management on macrovascular events and risk factors in the diabetes control and complications trial. Am J Cardiol 1995:75:894-903.

8 UK Prospective Diabetes Study (UKPDS) Group. Intensive blood-glucose control with sulphonylureas or insulin compared with conventional treatment and risk of complications in patients with type 2 diabetes (UKPDS 33). Lancet 1998;352:837-53.

- Main results of UKPDS comparing intensive and conventional treatment based on insulin or sulfonylureas.
4S DM

(placebo)

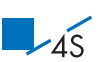

$14 \bar{S}$

(placebo)

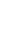

Figure 1 Evidence for the benefits of lipid lowering. Primary and secondary cholesterol lowering statin trials illustrating the percentage of patients with a coronary heart disease (CHD) event (cardiovascular death or non-fatal myocardial infarction) against the mean low density lipoprotein (LDL) cholesterol during the study in intervention and placebo groups. Adapted from LaRosa JC. Unresolved issues: unanswered questions. Eur Heart J 1999;1(suppl J): J18-23.

9 UK Prospective Diabetes Study (UKPDS) Group. Effect of intensive bloodglucose control with metformin on complications in overweight patients with type 2 diabetes (UKPDS 34). Lancet 1998;352:854-65.

- UKPDS subgroup of overweight patients (> $120 \%$ ideal body weight) treated with metformin.

10 Haffner SM, Greenberg AS, Weston WM, et al. Effect of rosiglitazone treatment on nontraditional markers of cardiovascular disease in patients with type 2 diabetes. Circulation 2002;106:679-84.

11 UK Prospective Diabetes Study Group. Tight blood pressure control and risk of macrovascular and microvascular complications in type 2 diabetes: UKPDS 38. BMJ 1998;317:703-13.

- UKPDS substudy in 1148 patients comparing less tight and tight blood pressure control based on atenolol or captopril.

12 Lindholm LH, Ibsen H, Dahlof B, et al. for the LIFE Study Group. Cardiovascular morbidity and mortality in patients with diabetes in the losartan intervention for endpoint reduction in hypertension study (LIFE): a randomised trial against atenolol. Lancet 2002;359:1004-10.

13 Parving $\mathrm{H}-\mathrm{H}$, Lehnert $\mathrm{H}$, Brochner-Mortensen J, et al. for the Irbesartan in Patients with Type 2 Diabetes and Microalbuminuria Study Group. The effect of irbesartan on the development of diabetic nephropathy in patients with type 2 diabetes. N Engl J Med 2001;345:870-8.

14 Lewis EJ, Hunsicker LG, Clarke WR, et al. for the Collaborative Study Group. Renoprotective effect of the angiotensin-receptor antagonist irbesartan in patients with nephropathy due to type 2 diabetes. N Engl J Med 2001;345:851-60.

15 Brenner BM, Cooper ME, de Zeeuw D, et al. for the RENAAL Study Investigators. Effects of losartan on renal and cardiovascular outcomes in patients with type 2 diabetes and nephropathy. N Engl J Med 2001;345:861-9.

16 The ALLHAT Officers and Coordinators for the ALLHAT Collaborative Research Group. Major outcomes in high-risk hypertensive patients randomized to angiotensin-converting enzyme inhibitor or calcium channel blocker vs diuretic. The antihypertensive and lipid-lowering treatment to prevent heart attack trial (ALLHAT). JAMA 2002;288:2981-97.

17 Heart Outcomes Prevention Evaluation (HOPE) Study Investigators. Effects of ramipril on cardiovascular and microvascular outcomes in people with diabetes mellitus: results of the HOPE study and MICRO-HOPE substudy. Lancet 2000;355:253-9.

18 Gerstein HC, Man JFE, Yi Q, et al. for the HOPE Study Investigators. Albuminuria and risk of cardiovascular events, death, and heart failure in diabetic and nondiabetic individuals. JAMA 2001;286:421-6.

19 Mann JFE, Gerstein HC, Pogue J, et al. for the HOPE Investigators. Renal insufficiency as a predictor of cardiovascular outcomes and the impact of ramipril: the HOPE randomized trial. Ann Intern Med 2001;134:629-36.

20 Heart Protection Study Collaborative Group. MRC/BHF heart protection study of cholesterol lowering with simvastatin in 20536 high-risk individuals: a randomised placebo-controlled trial. Lancet 2002;360:7-22. 